\title{
Evaluation of Remote Collaborative Manipulation for Scientific Data Analysis
}

\author{
Cédric Fleury \\ Insa de Rennes \\ IRISA, UMR CNRS 6074 \\ UEB, Rennes, France \\ fleury@cs.unc.edu
}

\author{
Thierry Duval \\ Université de Rennes 1 \\ IRISA, UMR CNRS 6074 \\ UEB, Rennes, France \\ thierry.duval@irisa.fr
}

\author{
Valérie Gouranton \\ Insa de Rennes \\ IRISA, UMR CNRS 6074 \\ UEB, Rennes, France \\ valerie.gouranton@irisa.fr
}

\author{
Anthony Steed \\ Department of \\ Computer Science \\ University College London \\ A.Steed@cs.ucl.ac.uk
}

\begin{abstract}
In the context of scientific data analysis, we propose to compare a remote collaborative manipulation technique with a single user manipulation technique. The manipulation task consists in positioning a clipping plane in order to perform cross-sections of scientific data that show several points of interest located inside these data. For the remote collaborative manipulation, we have chosen to use the 3-hand manipulation technique proposed by Aguerreche et al. [1], which is very suitable with a remote manipulation of a plane. We ran two experiments to compare the two manipulation techniques with some participants located in two different countries. These experiments has shown that the remote collaborative manipulation technique was significantly more efficient than the single user manipulation when the 3 points of interest were far apart inside the scientific data and, consequently, when the manipulation task was more difficult and required more precision. When the 3 points of interest were close together, there was not significant difference between the two manipulation techniques.
\end{abstract}

\section{Categories and Subject Descriptors}

H.5.3 [Informations Interfaces and Presentation]: Group and Organization Interfaces - Evaluation/methodology, Computer-supported cooperative work, Synchronous interaction; H.5.1 [Informations Interfaces and Presentation]: Multimedia Information Systems-Artificial, augmented, and virtual realities

\section{General Terms}

Experimentation, Human Factors, Performance

\section{Keywords}

Scientific data analysis, Remote collaborative manipulation, Virtual environment, VR experiments

Permission to make digital or hard copies of all or part of this work for personal or classroom use is granted without fee provided that copies are not made or distributed for profit or commercial advantage and that copies bear this notice and the full citation on the first page. To copy otherwise, to republish, to post on servers or to redistribute to lists, requires prior specific permission and/or a fee.

VRST'12, December 10-12, 2012, Toronto, Ontario, Canada.

Copyright 2012 ACM 978-1-4503-1469-5/12/12 ...\$15.00.

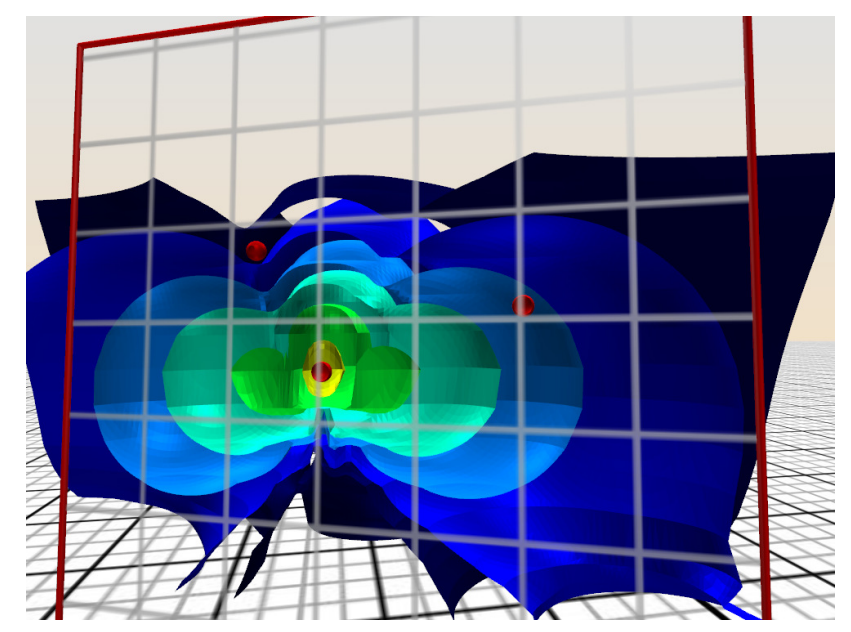

Figure 1: Cross-section of scientific data that shows the 3 points of interest (red spheres).

\section{INTRODUCTION}

Virtual reality is often used to visualise and to interact with scientific data. However, analysing scientific data can be a difficult task and it requires, sometimes, the knowledge of several remote experts. Distributed virtual reality enables remote experts to meet themselves in a virtual environment to perform joint review of scientific data. Most of the time, this joint review is limited to a simple observation of the scientific data and, when the users can interact, interactions are only sequential (just one user can access to tools at the same time). However, we think that collaborative interactions (parallel access to tools) could be useful for these experts to analyse together scientific data. On the one hand, if they are able to act together, it will increase their involvement in the task and their understanding of the data analysis. On the other hand, it can be helpful to perform some difficult manipulation tasks that require a good precision. So we propose to compare a remote collaborative manipulation technique with a single user manipulation technique for precisely positioning a clipping plane used to perform cross-sections of scientific data (see figure 1).

In this paper, we describe two experiments realised in order to compare these two kinds of manipulation techniques. For the single user manipulation, we have used a classical six degrees of freedom manipulation technique and, for the remote collaborative manipulation, we have chosen to use the 3-hand manipulation technique proposed by Aguerreche et 
al. [1]. Together the two experiments help us to determine situations when collaborative manipulation is useful.

This paper starts with an overview of related work focusing on scientific data analysis and collaborative interactions. It is followed by a description of the experimental context and the two manipulation techniques used. Part 4 presents the two experiments, their results, and a general discussion on these results. Then, the paper ends with a conclusion on this study and some perspectives for future experiments.

\section{RELATED WORK}

Lots of previous works use virtual reality for visualising scientific data as stated by Bryson [3]. Part 2.1 presents some applications in which a user analyses scientific data by making cross-sections of the data and some collaborative visualisation applications. Even if some visualisation applications enable several users to visualise together scientific data, none of them propose collaborative manipulations for exploring the data. Part 2.2 analyses the existing techniques for remote collaborative manipulation in virtual reality according to the requirements of the clipping plane manipulation.

\subsection{Scientific Data Analysis}

Several VR applications enable users to make cross-sections of scientific data. Hinckley et al. [8] propose to use a head and a cutting plane props to intuitively make a crosssection of brain MRI data. A props-based interaction device called the "cubic mouse" is also used by Fröhlich et al. [7] to position 3 orthogonal sections in geo-scientific data. Moreover, the 3D Sketch Slice [14] uses a tracked tablet to enable a user to control the position of a slice of seismic data using a 6 degrees of freedom manipulation. The tablet makes also possible to add some annotations inside the data. Even if these applications propose interesting techniques to make cross-sections of scientific data, none of them enables several users to make it collaboratively.

Leigh et al. [9] describe a range of examples of collaborative visualisation applications using immersive devices, but most of the time the users just observe the data and the only collaborative interaction consists in showing something to the others. Steed et al. [15] propose a interactive and collaborative system for the visualisation of medical data. Users can directly interact with the visualisation in order to drive an offline computation of the medical data. However, each user interacts alone and there is no collaborative manipulation.

\subsection{Collaborative Manipulation}

There are two categories of techniques that allow remote users to manipulate together a virtual object jointly: the techniques that split the degrees of freedom of a virtual object and the techniques that manage the concurrent access to the same degrees of freedom of a virtual object.

\subsubsection{Splitting the degrees of freedom}

As proposed by Pinho et al. [10], each user can control only some particular degrees of freedom of the virtual object. For example, one user can control the translation while another one can control the rotation. To perform this kind of manipulation, each user can use a particular tool more relevant for the degrees of freedom that he controls. For example, one user can use a virtual ray to move a virtual object while another one can use a virtual hand to rotate it and translate it along the virtual ray. However, this kind of collaborative manipulation is not relevant for the clipping plane manipulation because the users have very asymmetric role and none of the users can focus his action on finding particular points of interest without help of the others.

\subsubsection{Managing concurrent access to the same DOF}

Several techniques can be used to combine actions of several users to manipulate a virtual object as stated by Ruddle et al. [13]. A first solution consists in adding actions of these users, a second solution is to average their actions and a third solution proposes to keep only the common part of the actions (intersection). However, none of these solutions are ideal for the clipping plane manipulation because, if the users want to explore different parts of the data, they may perform contrary actions, which could disturb the others.

The Bent Pick Ray [11] technique enables several users to simultaneously manipulate a virtual object using virtual rays. This technique merges actions of all users by interpolating translations and rotations provided by the virtual rays. These virtual rays are also deformed according to their action on the virtual object to enable the users to understand the action of each user. This technique may be close to the average technique and the use of virtual rays does not seem to be very convenient for the clipping plane manipulation.

The SkeweR [5] technique enables two users to simultaneously grab any part of a virtual object. This technique determines the translation and the rotation of this grabbed object according to the positions of the 2 "grabbing points". A similar technique is used in a collaborative experiment that aims to construct a virtual gazebo [12]. Two users can manipulate a beam by grabbing its extremities using 2 virtual hands (one for each user). When the beam position cannot be resolved because the positions of the 2 virtual hands are not consistent, a red line is displayed between the beam and each virtual hands to show that the virtual hands are too far from the beam. However, these two techniques do not propose a solution to determine the rotation along the axis defined by the 2 "grabbing points". Since the clipping plane has to be rotated along each axis, these techniques seem not to be relevant for the clipping plane manipulation.

To avoid rotation issues, Aguerreche et al. [1] propose to add a third control point to manipulate virtual objects. In this 3-hand manipulation technique, virtual objects can be manipulated by 2 or 3 users together: either one user manipulates 2 control points and another one manipulates the third one, or each of 3 users manipulates one control point. The 3 translations of control points are sufficient to determine the resulting 6 degrees of freedom motion of manipulated objects. This technique coupled with a tangible interface (the 3 control points are attached to a tangible device) has been compared with the mean technique and the separation of degrees of freedom [2]. This evaluation has shown that the 3 -hand manipulation technique (using a tangible interface) is more accurate, more realistic and preferred by users. But, the technique had not yet been evaluated with remote users. We propose to use this technique for the remote collaborative manipulation of the clipping plane, because it is particularly well adapted to a precise manipulation of a plane (the positions of the 3 control points define a plane that can be the clipping plane). 


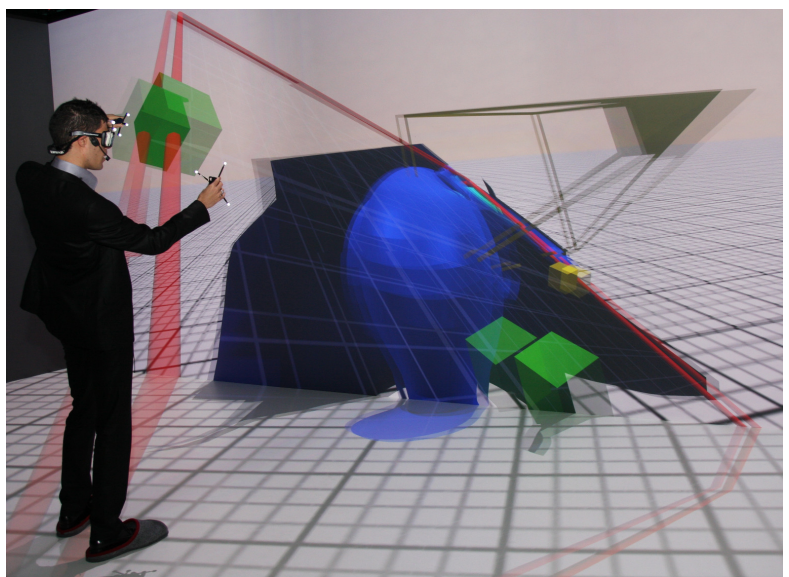

(a)

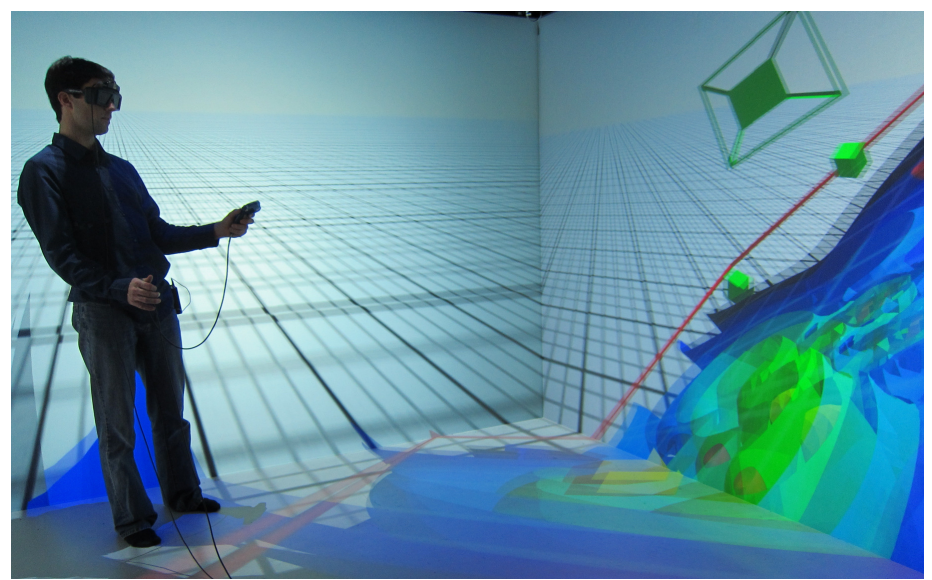

(b)

Figure 2: Collaborative manipulation of the clipping plane with the 3-hand manipulation technique : (a) the participant located in Rennes manipulated 2 control points (green cubes) and (b) the participant located in London manipulated one control point (yellow cube on the floor).

\section{CONTEXT}

During the experiments, participants had to manipulate a clipping plane that can be used to interactively perform cross-sections of scientific data. To manipulate this clipping plane, participants used either a single user manipulation technique or a collaborative manipulation technique which involved another remote participant. To avoid disturbing the manipulation task with a navigation task, navigation (locomotion) was turned off. However, users were tracked inside the immersive device and they could move in the virtual environment by using their physical movements.

\subsection{Single User Manipulation}

For the single user manipulation, a participant manipulated alone the clipping plane using a target tracked by a tracking system. The target was directly attached to the centre of the clipping plane (see figure 3 ). The participant could thus apply translations and rotations to the centre of the clipping plane with 6 degrees of freedom. This kind of manipulation was similar to the slice manipulation using a tracked tablet in the 3D Sketch Slice [14] application.

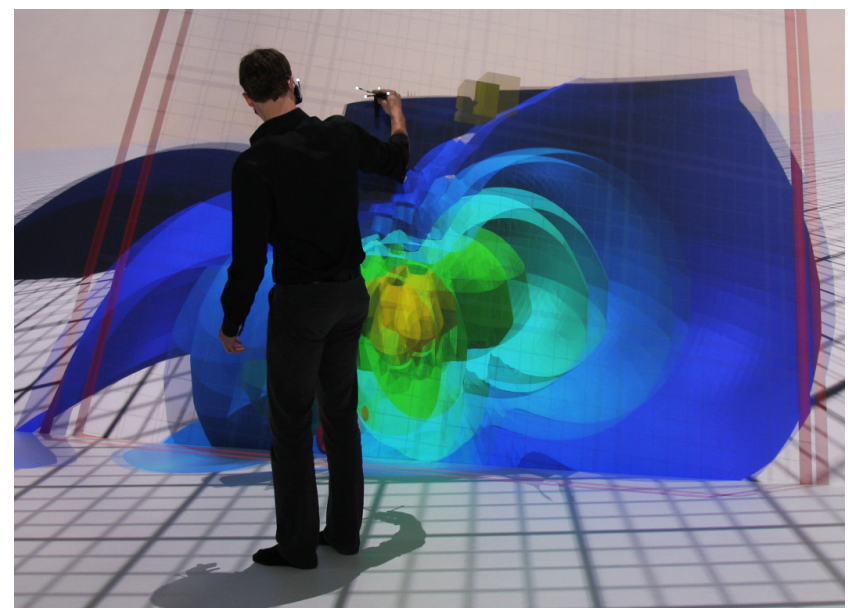

Figure 3: Single user manipulation of the centre of the clipping plane (yellow cube).

\subsection{Collaborative Manipulation}

For the collaborative manipulation, we have chosen to use the 3-hand manipulation technique proposed by Aguerreche et al. [1]. The participant located in Rennes manipulated 2 control points, using his two hands tracked by a tracking system. The participant located in London manipulated one control points, using one of his hands tracked by a tracking system. The positions of these 3 control points ( 2 in Rennes, 1 in London) defined the position of the clipping plane. So these control points enabled the two participants to move together the clipping plane (see figure 2).

Each participant was represented in the virtual world by his viewing frustum to enable the other to understand what he was seeing. His control point(s) used to manipulate the clipping plane was(were) also represented in the virtual world to enable the other to understand what he was doing (see figure 2). Moreover, the two participants could communicate by the voice with a microphone put on each participant and some speakers located in each immersive device.

\subsection{Technical Specifications}

To realise these experiments, a collaborative virtual environment has been deployed between Rennes and London. We first describe the immersive device used on each site, then we explain how the data of the virtual environment have been distributed for the collaborative manipulation.

\subsubsection{Immersive Devices}

On each side, participants used a specific immersive device to manipulate the clipping plane:

- In Rennes: the VR room is composed of 2 big screens (9,6 by $3 \mathrm{~m})$ with a resolution of $6240 \times 2016$ for the front and $3502 \times 1050$ for the floor. The front screen is back-projected by 8 projectors controlled by 2 Nvidia ${ }^{\circledR}$ Quatro ${ }^{\circledR}$ Plex 2200, while the floor is projected from above by 3 projectors controlled by 1 Nvidia ${ }^{\circledR}$ Quatro $^{\circledR}$ Plex 2200. An ART tracking system based on infrared cameras is used to track the user's head and hands inside the parallelepiped defined by the 2 screens.

- In London: the VR room is composed of 4 big screens (3 by $2,1 \mathrm{~m}$ ) with a resolution of $1400 \times 1050$ each (front, 
right, left and floor). Each of the front, right and left screens are back-projected by 1 projector controlled by a separate computer, while the floor is projected from above by 1 projector controlled by a 4 th computer. An InterSense tracking system based on ultra-sonic sensors is used to track the user's head and one of his hands inside the parallelepiped defined by the 4 screens.

On each side, the $j$ Reality graphical library [16] was used to render the virtual environment. This library makes possible to distribute the scene graph on the different computers managing the projectors of each VR room, to display stereoscopic images, and to deform the user's viewing frustum according to his head position (head tracking).

\subsubsection{Network Distribution}

For the single user manipulation, the data of the virtual environment were managed locally on each side. But, for the collaborative manipulation, these data were distributed on the network. We used a client/server architecture with a server located in Rennes. The two clients (one in Rennes, one in London) were connected to this server using TCP connections. The network latency between the server in Rennes and the client in London could be up to $50 \mathrm{~ms}$. We used the model proposed by Fleury et al. [6] to distribute the data of the virtual environment between the server and the two clients. This model makes it possible to choose a particular data distribution for each virtual object. We chose to process the scientific data, the points of interest, the clipping plane and the viewing frustum representations on the server to ensure a strong consistency between the two participants' view. However, we chose to process the control points of the clipping plane on each client to ensure a good responsiveness when the participants were moving these points.

With this data distribution, a small gap could occur between the control points and the clipping plane due to the network latency. However, it was less disturbing for the participants that this gap occurred between the control points and the clipping plane than between the participant real hands and the control points. Moreover, it occurred only when the participants moved very quickly the clipping plane and not when they moved slowly the clipping plane to perform precise manipulation tasks. None of the participants complained about this small gap during the experiments.

\section{EXPERIMENTS}

We ran two experiments to compare the single user manipulation with the remote collaborative manipulation of the clipping plane. Even if the first experiment has not shown significant difference between the two manipulation techniques, the observations performed during this experiment have allowed us to formulate two interesting new hypotheses. The second experiment aimed to validate these hypotheses by modifying the experimental conditions.

\subsection{First Experiment}

To compare the two manipulation techniques for scientific data analysis, we ran a first experiment in which the participants had to examine some real scientific data.

\subsubsection{Description}

The scientific data were seismic data from a physical simulation of an earthquake near Nice in France [4]. This earthquake is not real, but it is realistic according to localisation and intensity. Data used in the experiment were iso-surfaces of the PGD (Peak Ground Displacement) computed during the simulation. The iso-surfaces were organised on a concentric way around the earthquake's epicentre (see figure 1).

\section{Task to Perform.}

Participants had to find 3 points of interest inside the scientific data. For the experiments, these points of interest were represented by small red spheres. To find the points of interest, participants manipulated the clipping plane using either the single user manipulation (part 3.1) or the collaborative manipulation with a remote participant (part 3.2). When participants had found the 3 points of interest, they had to precisely move the clipping plane in order to reach the 3 points at the same time and to make a cross-section that showed the 3 points at the same time (see figure 1 ).

\section{Population.}

10 participants in Rennes ( 1 female and 9 males) aged from 20 to 39 (mean: 24, standard deviation: 5,63) took part in this experiment. They performed the collaborative manipulation with a confederate in London (always the same co-author). One of two participants performed first the single user manipulation and then the collaboration manipulation, and vice versa for the other half of the participants.

\section{Procedure.}

After a training period, each participant performed 5 trials for each manipulation technique. For each trial, the positions of the 3 points of interest were randomly chosen in a set of 5 interesting configurations of the points. When one configuration was chosen for a trial, it was removed from the set. The same set of configurations was used for each of the two techniques and for each participant.

\section{Collected Data.}

For each trial and each participant, we recorded the completion time. Time recording started when the participant activated the manipulation of the clipping plane by pressing a control button, and was automatically stopped when the clipping plane reached the 3 points at the same time.

\subsubsection{Results}

This experiment showed that the difference between the two manipulation techniques was not significant. First, the mean completion times of all the participants for the two techniques were very close: $71.66 \mathrm{sec}$ with the single user manipulation and $67.66 \mathrm{sec}$ with the collaborative manipulation. Second, there was an important participant variability: standard deviation of the mean completion time was equal to $89.14 \mathrm{sec}$ for the single user manipulation and to $32.49 \mathrm{sec}$ for the collaborative manipulation. A Student's test (t-test) showed that the difference between the two techniques was not significant $(t(18)=0.13, p=0.9)$. This not significant difference can be explained by the following observations:

1. The distance between the 3 points of interest was too small (and stayed always similar on each trial). Indeed, for some participants, the task to complete was very easy and took them just few seconds. So, for these participants, it was difficult to make a difference between the two manipulation techniques. Moreover, if the task is too easy, the time required by the two par- 
ticipants to synchronise themselves at the beginning of a collaborative manipulation penalises this technique.

2. The search of the 3 points of interest inside the scientific data introduced a bias in the evaluation of the two manipulation techniques. Indeed, the participants did not have particular knowledge in analysing scientific data and, for some of them, it was very difficult to find the 3 points of interest inside the data. For these participants, it was impossible to compare the two techniques together, because the completion time depended more on the "luck" of finding quickly the points of interest than on the time required to well adjust the clipping plane position.

3. The training period was maybe too short. Indeed, we noticed that the completion time was shorter for the last trials than for the first trials. It was even more noticeable for the collaborative manipulation technique.

Observations 2 and 3 have been identified as sources of bias that required to be controlled in next experiment, while first observation could be used to formulate two hypotheses:

- H1: when the 3 points of interest are close together (and the task is almost easy), the single user manipulation is as efficient as the collaborative manipulation.

- H2: if the distance between the 3 points of interest increases (and thus the task becomes more difficult), then the collaborative manipulation will be more efficient than the single user manipulation.

\subsection{Second Experiment}

To test the hypothesis $\mathbf{H 1}$ and $\mathbf{H 2}$ formulated in the first experiment, we have performed a second experiment by adapting the experimental protocol.

\subsubsection{Description}

First, to avoid the bias described in the observations 2 and 3 , we have decided, respectively, to remove the scientific data in order to not mix the manipulation task with a search task, and to increase the training period for the two manipulation techniques. Second, we have decided to keep some similar configurations of the 3 points of interest to test $\mathbf{H 1}$, and to introduce some new configurations with a significantly bigger distance between the 3 points of interest to test $\mathbf{H 2}$.

\section{Task to Perform.}

Even if the scientific data had been removed, participants still had to manipulate a clipping plane in order to reach 3 points of the virtual world at the same time (see figure 4). To manipulate this plane, participants used either the single user manipulation (part 3.1) or the collaborative manipulation with a remote participant (part 3.2). Some new configurations of the 3 points with a bigger distance between the points had been added, and all the configurations used for the experiment had been divided into two groups according to the distance between the points (mean of the distances two by two between the 3 points):

- a "Close" group with the configurations for which the mean of the distances was less than $0,6 \mathrm{~m}$,

- a "Far" group with the configurations for which the mean of the distances was more than $1,4 \mathrm{~m}$.

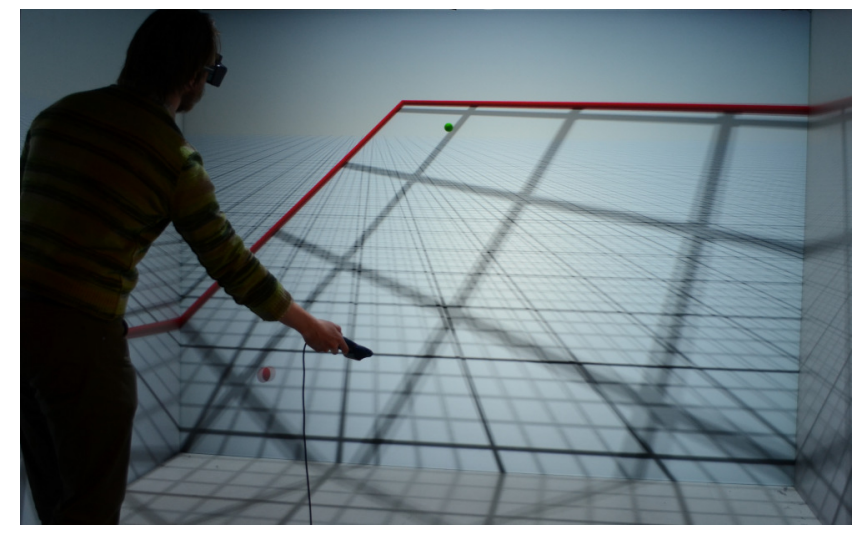

Figure 4: 2nd experiment that consisted in manipulating the clipping plane without the scientific data.

\section{Population.}

32 participants ( 6 females and 26 males) aged from 18 to 50 (mean: 26, standard deviation: 6,72) took part in this experiment. None of these participants had been involved in the first experiment. They were divided in two groups:

- a group G1 of 16 participants in Rennes who performed the collaborative manipulation with a confederate in London (always the same co-author). Only the 16 participants in Rennes performed the single user manipulation (the confederate did not perform the single user manipulation), and 16 teams (each participant in Rennes with the confederate in London) performed the collaborative manipulation.

- a group G2 of 16 participants (8 in Rennes, 8 in London) who performed the collaborative manipulation with another real participant of this group. The 16 participants, both in Rennes and London, performed separately the single user manipulation, but only 8 teams (one participant in Rennes with one participant in London) performed the collaborative manipulation.

In each group, one of two participants performed first the single user manipulation and then the collaboration manipulation, and vice versa for the other half of the group.

\section{Procedure.}

After time to familiarise themselves with the virtual environment, participants realised first a training for the two techniques in the same order than for the real experiment. The training consisted in performing 4 trials for each technique. When participants had finished the training, they started the real experiment. They had to performed 10 trials for each manipulation technique: 5 used a configuration of 3 Close points and 5 used a configuration of 3 Far points. The 5 trials with the Close points were randomly mixed with the 5 trials with the Far points. The experiment lasted about 45 minutes including training trials.

\section{Collected Data.}

For each trial and each participant, we recorded the completion time in the same way as in the first experiment. After the experiment, participants filled out a subjective questionnaire about the two manipulation techniques. Obviously, the confederate did not fill out this questionnaire. 


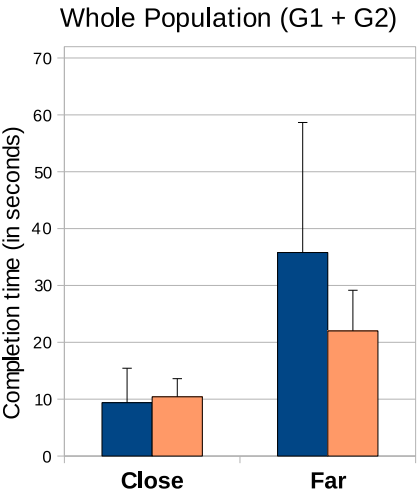

(a)

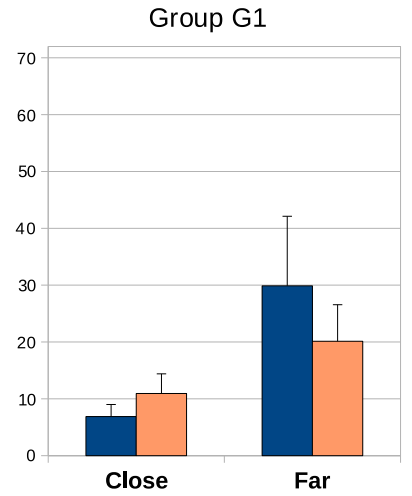

(b)

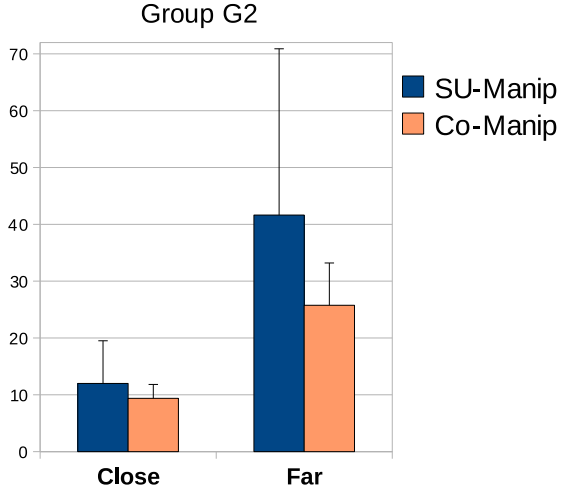

(c)

Figure 5: Means and standard deviations of the completion time for the two techniques for the whole population (a), for group G1 (b) and for group G2 (c).

\subsubsection{Results}

Using the data collected during the experiment, we conducted statistic analysis to compare the single user manipulation (SU-Manip) with the collaborative manipulation (CoManip). For these statistic analysis, we separated the cases when the 3 points were Close together and the cases when the 3 points were Far apart.

\section{Completion Time.}

For each participant, we computed the mean completion time on the 5 trials for each group of points and for each manipulation technique (4 cases). Then, we performed a Student's test (t-test) on these mean values (in seconds) to compare the two techniques (SU-Manip, Co-Manip). We also computed the mean values $(M)$ and the standard deviation $(S D)$ of the mean completion times of all the participant, for each one of the 4 cases.

First, we considered the whole population $\mathbf{G 1}+\mathbf{G} 2$ (see figure $5(\mathrm{a}))$. When the 3 points were Close together, the mean completion time was almost the same with the SUManip technique $(M=9.38 \mathrm{sec}, S D=6.06 \mathrm{sec})$ and with the Co-Manip technique $(M=10.42 \mathrm{sec}, S D=3.19 \mathrm{sec})$, and the difference between both techniques was not significant $(t(54)=-0.76, p=0.44)$. However, when the 3 points were Far apart, the mean completion time was shorter with the Co-Manip technique $(M=22 \mathrm{sec}, S D=$ $7.15 \mathrm{sec})$ than with the SU-Manip technique $(M=35.78 \mathrm{sec}$, $S D=22.88 \mathrm{sec})$, and this difference was significant $(t(54)=$ $2.84, p=0.006)$.

Second, we only considered the group G1 of the participants who had performed the Co-Manip with the same confederate in London (see figure 5(b)). When the 3 points were Close together, the mean completion time was slightly shorter with the SU-Manip technique $(M=6.88 \mathrm{sec}, S D=$ $2.13 \mathrm{sec})$ than with the Co-Manip technique $(M=10.94 \mathrm{sec}$, $S D=3.45 \mathrm{sec})$, and this difference was significant $(t(30)=$ -44.01, $p<0.001)$. When the 3 points were Far apart, the mean completion time was shorter with the Co-Manip technique $(M=20.13 \mathrm{sec}, S D=6.43 \mathrm{sec})$ than with the SU-Manip technique $(M=29.88 \mathrm{sec}, S D=12.25 \mathrm{sec})$, and this difference was significant $(t(30)=2.82, p=0.008)$.

Finally, we only considered the group G2 of the par- ticipants who had performed the Co-Manip with another real participant of the group (see figure 5(c)). When the 3 points were Close together, the mean completion time was slightly shorter with the Co-Manip technique $(M=9.38 \mathrm{sec}$, $S D=2.45 \mathrm{sec})$ than with the SU-Manip technique $(M=$ $12 \mathrm{sec}, S D=7.5 \mathrm{sec}$ ), but this difference was not significant $(t(22)=0.96, p=0.35)$. When the 3 points were Far apart, the mean completion time was shorter with the Co-Manip technique $(M=25.75 \mathrm{sec}, S D=7.44 \mathrm{sec})$ than with the SUManip technique ( $M=41.63 \mathrm{sec}, S D=29.27 \mathrm{sec})$, however this difference was not significant $(t(22)=1.49, p=0.149)$. We thought that, even if there was a big difference between the mean completion time for the two techniques, this difference was not significant for the farther apart points because:

- Some participants had lot of difficulties to perform the task with the SU-Manip technique when the 3 points were Far apart (it could explain the big standard deviation for the Far points).

- Some two-person teams in the group G2 had difficulties to synchronise themselves at the beginning of the Co-Manip (language barrier, etc.). It induced some big differences between the completion time of each two-person team.

- In the group G2, each participant performed the task alone for SU-Manip (16 participants) and with another participant of the group for the Co-Manip (8 teams). Thus, the number of samples for the SU-Manip was twice the number of samples for the Co-Manip.

\section{Subjective Questionnaire.}

After the experiment, a preference questionnaire was proposed in which the participants had to grade from 1 to 7 (7point Likert scale) the two manipulation techniques for each group of point configurations (Close or Far) according to 5 subjective criteria: fatigue, ease of use, precision, naturalness and a global appreciation of the technique. Moreover, for the Co-Manip, they had to grade from 1 to 7 for each group of point configurations their feeling of collaborating with another real participant. We computed the mean values and the standard deviation on ratings given by the whole 


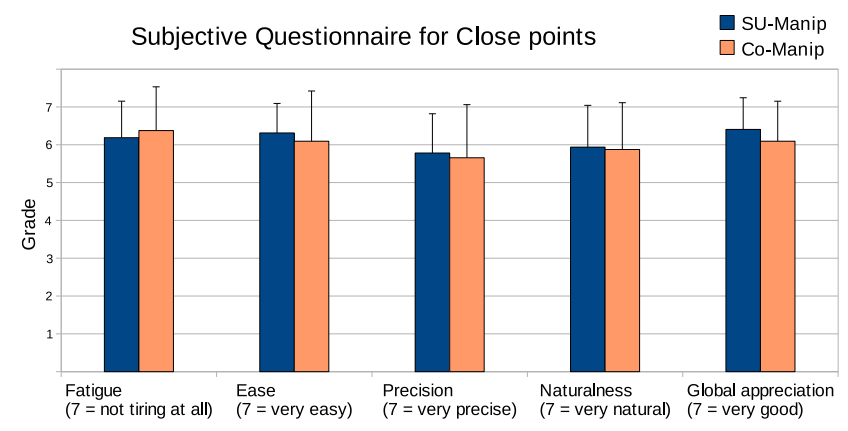

Figure 6: Means and standard deviations of subjective ratings for the two techniques when the 3 points were Close together.

population $\mathbf{G 1}+\mathbf{G 2}$ for each one of the 4 cases. Then, to compare the two manipulation techniques, we computed the differences $(\Delta)$ between the mean values of the two techniques and we performed a Wilcoxon signed rank test on ratings given by the participants to determine if these differences were significant.

First, we considered the subjective ratings given when the 3 points were Close together (see figure 6): it did not seem to have particular preferences for one or the other manipulation technique. Indeed, the differences between the mean values of the two techniques were very low and the statistical analysis showed that these differences were not significant: fatigue $(\Delta=0.19, p=0.21)$, ease $(\Delta=0.22, p=0.58)$, precision $(\Delta=0.13, p=0.77)$, naturalness $(\Delta=0.06, p=1)$ and global appreciation $(\Delta=0.31, p=0.08)$.

Second, we considered the subjective ratings given when the 3 points were Far apart (see figure 7): it seemed that the Co-Manip technique was more preferred than the SUManip technique. Indeed, the differences between the mean values of the two techniques were substantial (more than 1 point) for each criterion and for the global appreciation. The statistical analysis showed that these differences were highly significant: fatigue $(\Delta=1.44, p<0.001)$, ease $(\Delta=$ $2.19, p<0.001)$, precision $(\Delta=1.34, p<0.001)$, naturalness $(\Delta=1.31, p<0.001)$ and global appreciation $(\Delta=1.47, p<0.001)$.

Finally, whatever the distance between the 3 points, it seemed that the participants had a strong feeling of collaborating with another remote participant: for the Close points $(M=5.69, S D=1.18)$ and for the Far points $(M=6.34, S D=0.97)$. Moreover, it is interesting to notice that the feeling of collaboration was slightly stronger when the 3 points were Far apart and the statistic analysis showed that this difference was significant $(\Delta=0.66, p=0.007)$. We can think that the participants felt more the need to collaborate when the task to perform was more difficult (i.e. when the 3 points were farther apart).

\subsection{Discussion}

The results obtained in the second experiment showed that there was not a significant difference between the two manipulation techniques when the 3 points were closer together. Indeed, the completion time was shorter either with the single user manipulation technique or with the collaborative manipulation technique according to the studied popu-

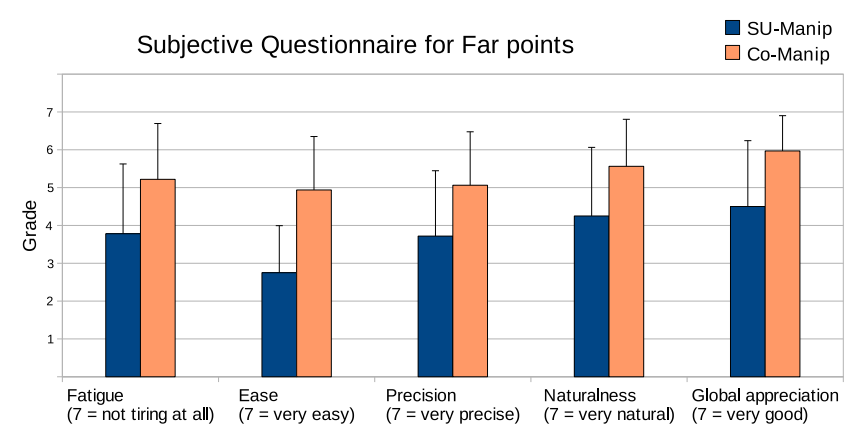

Figure 7: Means and standard deviations of subjective ratings for the two techniques when the 3 points were Far apart.

lation, and these differences were not significant. Moreover, the subjective questionnaire did not show particular preferences for one or the other manipulation technique when the 3 points were closer together. This lack of significant differences between the two techniques validated hypothesis $\mathbf{H 1}$ stated in the first experiment.

When the 3 points were far apart, the second experiment showed that a collaborative manipulation between two remote users was more efficient than a single user manipulation to analyse the scientific data. It can be explained by two factors: when the 3 points were farther apart, first, the task of positioning the clipping plane required more precision and, second, the participants could not see all the 3 points at the same time in their field of view and they had to rotate the head to adjust the clipping plane position. These two factors globally increased the difficulty of performing the task when the 3 points were farther apart. With the collaborative manipulation technique, each user could focus on adjusting the clipping position to reach only one or two points and let the other do the same for the other points. So, the completion time was significantly shorter with the collaborative manipulation technique than with the single user manipulation technique. Moreover, the subjective questionnaire showed that the participants globally preferred the collaborative manipulation technique when the 3 points were farther apart, and they also found this technique less tiring, easier, more precise and more natural for these point configurations. Consequently, hypothesis H2 stated in the first experiment was validated.

For the second experiment, there were not noticeable evolution of the completion time between the first trials and the last trials. So we could conclude that the observation 3 of the first experiment was true, and that the training period in the second experiment was sufficient. However, nothing enabled us to corroborate the observation 2 stated in the first experiment. It would be interesting to realise a new experiment with the scientific data and the farther apart points to determine if the presence of the scientific data impacts the manipulation task. Moreover, it could also be interesting to propose some solutions to enable participants to take advantage of the collaboration for searching the points of interest inside the data.

Finally, the less significant results obtained for the group G2 in the second experiment showed that collaborative experiments are difficult to design because so many parameters 
are involved. Indeed, when remote participants who do not know each other collaborate together, some additional difficulties can occur according to the participants' language, their vocabularies, their predispositions for the collaborative work or their goodwill to work with someone else.

\section{CONCLUSION}

These experiments aimed to compare a single user manipulation technique with a collaborative manipulation technique between two remote users (one located in Rennes and the other located in London) for analysing some scientific data. The task consisted in positioning a clipping plane in order to show, at the same time, 3 points of interest located inside the scientific data. For the single user manipulation, a participant manipulated alone the clipping plane by rotating and translating its centre (6 degrees of freedom). For the collaborative manipulation, two participants manipulated together the clipping plane by using the 3-hand manipulation technique proposed by Aguerreche et al. [1]. Even if there were not significant differences between the two manipulation techniques when the 3 points of interests were close together, the experiments showed that the remote collaborative manipulation was more efficient than the single user manipulation when the 3 points were far apart and, consequently, when the task to perform was more difficult.

In future work, we would like to perform a new experiment to determine the threshold of the distance between the points of interest from which it becomes more efficient to use the remote collaborative manipulation technique than the single user manipulation technique. It would also be interesting to run experiment with the scientific data and the farther apart points as explained in the discussion part. In this new experiment with scientific data and farther apart points, we guess that maybe the manipulation of a clipping plane could interfere with the individuals' visualisation of the scientific data. Indeed, the manipulation of one user could lead sometimes to "put" the other inside the data (by reversing the clipping plane for instance). In this case, the second user loses track of the points of interest on which he is focusing. There are thus some potential rendering challenges to address in order to allow this user to continue his interaction even if he is inside the scientific data.

\section{ACKNOWLEDGMENTS}

We wish to thank the Visionair European infrastructure project, the Foundation Rennes 1 "Progress, Innovation, Entrepreneurship" and the French Research National Agency project named Collaviz for their support.

\section{REFERENCES}

[1] L. Aguerreche, T. Duval, and A. Lécuyer. Short paper: 3-Hand Manipulation of Virtual Objects. In Proc. of the Joint Virtual Reality Conf. of EGVE - ICAT Euro VR, pages 153-156, 2009.

[2] L. Aguerreche, T. Duval, and A. Lécuyer. Evaluation of a Reconfigurable Tangible Device for Collaborative Manipulation of Objects in Virtual Reality. In Proceedings of Theory and Practice of Computer Graphics Conference, pages 81-88, 2011.

[3] S. Bryson. Virtual reality in scientific visualization. Communications of the ACM, 39(5):62-71, may 1996.
[4] F. Dupros, F. D. Martin, E. Foerster, D. Komatitsch, and J. Roman. High-performance finite-element simulations of seismic wave propagation in three-dimensional nonlinear inelastic geological media. Parallel Computing, 36(5-6):308-325, 2010.

[5] T. Duval, A. Lecuyer, and S. Thomas. SkeweR: a 3D Interaction Technique for 2-User Collaborative Manipulation of Objects in Virtual Environments. In Proceedings of the IEEE symposium on $3 D$ User Interfaces, pages 69-72, 2006.

[6] C. Fleury, T. Duval, V. Gouranton, and B. Arnaldi. A New Adaptive Data Distribution Model for Consistency Maintenance in Collaborative Virtual Environments. In Proc. of the Joint Virtual Reality Conf. of EuroVR - EGVE - VEC, pages 29-36, 2010.

[7] B. Fröhlich, S. Barrass, B. Zehner, J. Plate, and M. Göbel. Exploring geo-scientific data in virtual environments. In Proceedings of the conference on Visualization, pages 169-173, 1999.

[8] K. Hinckley, R. Pausch, J. C. Goble, and N. F. Kassell. Passive real-world interface props for neurosurgical visualization. In Proceedings of the SIGCHI conference on Human factors in computing systems, pages 452-458, 1994.

[9] J. Leigh, A. E. Johnson, M. Brown, D. J. Sandin, and T. A. DeFanti. Visualization in Teleimmersive Environments. Computer, 32(12):66-73, Dec. 1999.

[10] M. S. Pinho, D. A. Bowman, and C. M. Freitas. Cooperative object manipulation in immersive virtual environments: framework and techniques. In Proc. of the ACM symposium on Virtual Reality Software and Technology, pages 171-178, 2002.

[11] K. Riege, T. Holtkamper, G. Wesche, and B. Frohlich. The Bent Pick Ray: An Extended Pointing Technique for Multi-User Interaction. In Proc. of the IEEE symp. on $3 D$ User Interfaces, pages 62-65, 2006.

[12] D. Roberts, R. Wolff, O. Otto, and A. Steed. Constructing a Gazebo: supporting teamwork in a tightly coupled, distributed task in virtual reality. Presence: Teleoperators and Virtual Environments, 12(6):644-657, Dec. 2003.

[13] R. A. Ruddle, J. C. D. Savage, and D. M. Jones. Symmetric and asymmetric action integration during cooperative object manipulation in virtual environments. ACM Transaction Computer-Human Interaction, 9(4):285-308, Dec. 2002.

[14] J. Schild, T. Holtkämper, and M. Bogen. The 3D Sketch Slice: Precise 3D Volume Annotations in Virtual Environments. In Proceedings of the Joint Virtual Reality Conference of EGVE - ICAT Euro VR, pages 65-72, 2009.

[15] A. Steed, D. Alexander, P. Cook, and C. Parker. Visualizing Diffusion-Weighted MRI Data Using Collaborative Virtual Environment and Grid Technologies. In Proceedings of the Theory and Practice of Computer Graphics, pages 156-161, 2003.

[16] S. Weißmann, C. Gunn, P. Brinkmann, T. Hoffmann, and U. Pinkall. jReality: a java library for real-time interactive 3D graphics and audio. In Proc. of the ACM conference on Multimedia, pages 927-928, 2009. 